\title{
An Overview of Application of Artificial Immune System in Swarm Robotic Systems
}

\section{Daudi J*}

Department of Aerospace Engineering, School of Engineering, University of Glasgow, UK

\begin{abstract}
The Artificial Immune System (AIS) is a biologically inspired computation system based on specifically human immune system. AIS applications in last one decade have been developed to address the complex computational and engineering problems related to classification, optimisation and anomaly detection. Many investigations have been conducted to understand the principles of immune system to translate the knowledge into AIS applications. However, a clear understanding of principles and responses of immune system is still required for application of AIS to Swarm Robotics. This article after a review of AIS models and algorithms proposes an integration of AIS and Swarm Robotics by developing a very clear understanding of immune system structures and associated functions.
\end{abstract}

Keywords: Immune System (IS); Artificial immune systems (AIS); Artificial Immune Algorithms; Neutrophils; Swarm Robotics (SR)

\section{Introduction}

Man has survived through millions of year and the credit goes to the natural defense system our bodies are blessed with, the "Immune System (IS)". The immune system provides protection to a living body against number for foreign molecules (referred to as antigens) e.g. viruses, bacteria, fungi and other parasites. The immune system achieve this objective by observing, studying and identifying foreign molecules that enter our bodies, then, it prompts its response against them by creating and releasing antibodies that attack these antigens, thus eliminating them from our bodies and freeing us from infections. To eliminate the threat, IS has to distinguish between foreign molecules and the molecules/tissues that constitute itself to avoid auto-immune responses.

Immune System possesses excellent ability to recognize the foreign molecules, when they are encountered for the very first time, retain their memory and identify them when encountered at a later stage. IS uses genetic characteristics for biological functioning and thus provide the base for computational modelling of adaptive or inborne responses. Following human central nervous system; IS is the most complex biological system due to wider and variable responses. Based on its complex and multiple behaviours, its understanding for computational adoption is still inadequate.

\section{Types of Immune System}

Immune system is sub-divided into two types of systems i.e. adaptive and innate immunity.

Adaptive immunity is directed against specific disease causing foreign agents and is modified by exposure to such organisms or antigens and keeps strong immunological memory. Adaptive immunity targets the specific pathogens, either previously encountered or not and gets modified by exposure to such pathogens. Adaptive immunity system consists of lymphocytes (white blood cells, more specifically $\mathrm{B}$ and $\mathrm{T}$ type) which function to recognize and destroy specific substances, and are antigen-specific [1]. Adaptive immune system has Immunological memory which gives the system, capability of more effective immune response against an antigen after its first encounter, leaving the body in a better position to be able to resist in the future against same pathogens. On primary response, the immune system identifies an antigen and responds with release of large number of antibodies to fight with infection and to eliminate the antigen from the body.

Innate immunity is aimed to target any invaders or disease causing agents or pathogen in the body and is non-specific. It is not modified by repeated exposure and thus plays an important role in the initiation and regulation of immune responses. Innate immunity involves number of specialized white blood cells involved to recognize and bind to common molecular patterns of disease causing microorganisms. It does not provide complete protection and is primarily static in nature and does not modify [1]. The innate immune system is considered as the first line of defense [2] which comprised of cells and mechanisms that defend the host from infection by disease causing organisms. Unlike the adaptive immune system (which is found only in vertebrates), it does not confer long-lasting or protective immunity to the host [3]. Innate immune system recruits the immune cells to sites of infection, through the production of specialized chemical mediators, called cytokines. This causes the identification and removal of foreign substances infecting organs, tissues, blood and lymph vessels by specialised white blood cells. Generally, innate and adaptive immunity systems have following characteristics indicated as Figure 1.

All white blood cells (WBC) are known as leukocytes. Leukocytes are not associated with a particular organ or tissue; thus, they function like a unicellular organism. Leukocytes are able to move as singular cell or as a population of cells and respond against foreign agents. The innate leukocytes or white blood cells include: Natural killer cells, mast cells, eosinophils, basophils; and the phagocytic cells including macrophages, neutrophils, and dendritic cells.

Artificial Immune System is actually based upon the capabilities

*Corresponding author: Daudi J, Department of Aerospace Engineering, School of Engineering, University of Glasgow, UK, Tel: 44 (0) 141 330; E-mail: daudij@yahoo.com

Received January 21, 2015; Accepted February 05, 2015; Published February 14,2015

Citation: Daudi J (2015) An Overview of Application of Artificial Immune System in Swarm Robotic Systems. Adv Robot Autom 4: 127. doi:10.4172/2168 9695.1000127

Copyright: (c) 2015 Daudi J. This is an open-access article distributed under the terms of the Creative Commons Attribution License, which permits unrestricted use, distribution, and reproduction in any medium, provided the original author and source are credited. 


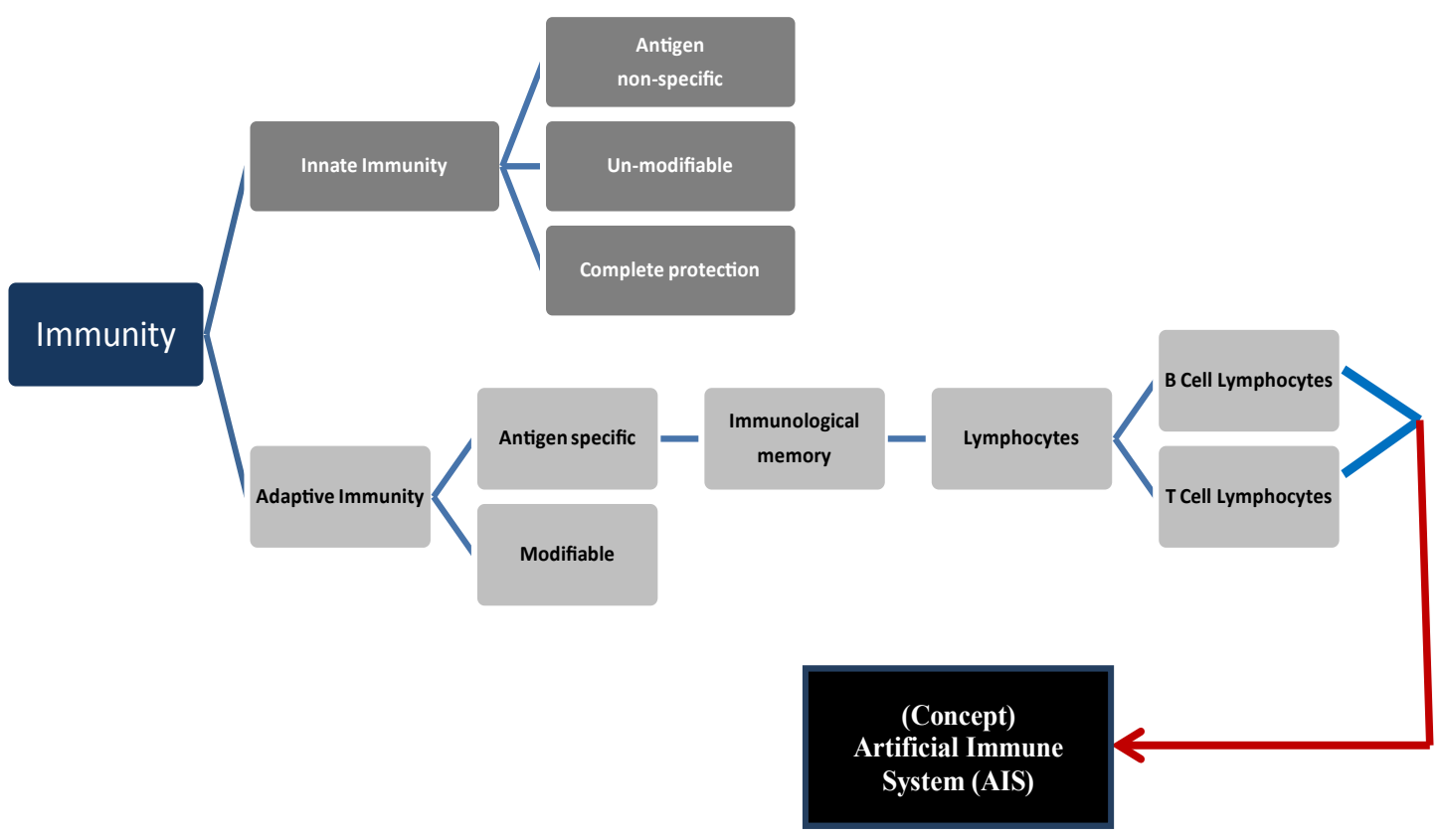

Figure 1: Classification of Human Immune System.

of immune system such as robustness, de-centralization, error tolerance and adaptiveness which enabled the researchers for system computation as "Artificial Immune System (AIS)" [4-6].

Following the patterns and behaviours of IS three basic computational areas are developed such as immune modelling, theoretical AIS and applied AIS. Immune modelling is focused on mathematical models and simulations of natural and artificial immune systems. Theoretical AISs concerned with the theoretical aspects including mathematical modelling of algorithms, convergence analysis, and performance and complexity analysis of such algorithms. Applied AISs includes working on immune-inspired algorithms, building immune-inspired computer systems, to apply AISs to diverse real world applications [7].

AIS follows the IS characteristics for computational application development such as feature extraction, pattern recognition, memory, learning, classification, adaption for utilization in computer security, fraud detection, machine learning, data analysis, optimization algorithms [8].

\section{AIS Algorithms}

A critical review of literature has concluded that four major AIS algorithms are focused to develop various AIS applications (Figure 2).

\section{Negative selection based algorithms}

Negative Selection Algorithms are based on the principle function that protects the body against self-reactive lymphocytes. IS identifies foreign antigens without reacting with the 'self cells'. Receptors are produced during a pseudo-random genetic re-arrangement process for the generation of T-cells. These receptors undergo negative selection mechanisms in thymus. Those T cells which react against "Self Cells" or against self-proteins are discarded and remaining which do not bind to self-cells are permitted to leave the thymus. These are called as matured T-cells which are allowed free movement throughout the body to

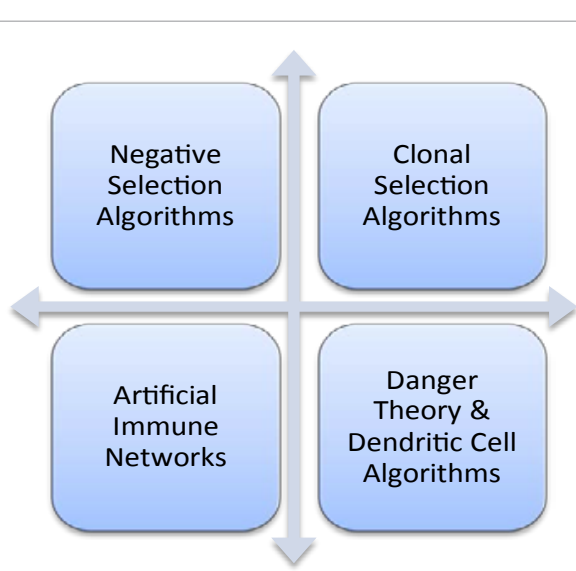

Figure 2: AIS Algorithms.

contribute in immunological process against foreign antigens [9]. This biological phenomenon has given an inspiration for the developments of most of the existing Artificial Immune systems.

\section{Clonal selection based algorithms}

This Clonal Selection Based Algorithms is formulated on the principle of mechanism of antigen-antibody recognition, binding, cell propagation and separation into memory cell [10]. This is called as Clonal Selection Theory which has resulted in development of several artificial immune algorithms named as clonal selection algorithm (CSA) by Castro and Zuben [11]. Based on clonal selection and affinity maturation principles they named this as CLONALG. CLONALG in 2002.

\section{Artificial immune networks (AINs)}

Artificial immune networks (AINs) are other successful models in AISs. Framer's etal proposed their immune network model Farmer 
et al. which became the fundamental for various AINs algorithm and hence based on this the first immune network algorithm was proposed by Ishida [12]. Later, Timmis et al. re-defined these immune networks, which were formally named as AINE (Artificial Immune Network). According to Knight and Timmis. 2001 AINs uses Artificial Recognition Ball (ARB) to represent identical B-cells [13]. Two B-Cells are joined together, if the affinity between two ARBs is below a network affinity threshold (NAT). A Resource Limited Artificial Immune System (RLAIS) based on AINE is developed in 2001 by Timmis and Neal. This up-gradation of models included the knowledge of the fixed total number of B-cells presented in ARBs with centralized control but having the specific role of each ARB in obtaining resources from the mainstream. Those ARBs not able to obtain resources are removed from the network. The cloning, Mutation and interactions of B-Cells all take place at the ARB level.

\section{Danger theory and dendritic cell algorithms}

Dendritic cells within an innate immune system are cells which respond to some specific danger signals. The three main types of dendritic cells such as: 1) Immature Dendritic Cells, which collect parts of the antigen and the signals, 2) Semi-mature Dendritic cells, are immature cells which have decision power against local signals and represent safe and present the antigen to T-cells resulting in tolerance, and 3) Mature Dendritic cells, are capable to react strongly identifying that the local signals represent danger and deliver the antigen to T-cells for reaction against pathogens. The Dendritic Cell Algorithm is biologically inspired development taking inspiration from the Danger Theory of the mammalian immune system with specific function of dendritic cells. Matzinger, first proposed this Danger Theory stating that the roles of the acquired immune system is to respond to signals of danger, rather than discriminating self from non-self $[14,15]$. The theory states that the helper T-cells activate an alarm signal providing the co-stimulation of antigen-specific cells to respond. The Dendritic Cell Algorithm (DCA) is inspired by the function of the dendritic cells of the human immune system.

An abstract model of dendritic cell (DC) behaviour was also developed and used to form an algorithm, the artificial DCA by Greensmith et al. For this purpose, population based DCA algorithm was applied to numerous intrusion detection problems in computer security including the detection of port scans and botnets, where it has produced impressive results with relatively low rates of false positives [16].

There are several other immunology areas reported in the literature to inspire the development of algorithms and computational tools, for example, humoral immune response, Danger Theory dendritic cell functions, and Pattern Recognition Receptor Model [17-20].

\section{Review of AIS Application Areas}

Dasgupta in 1999 under the title "Artificial Immune Systems and Their applications" compiled several useful literature resources including AIS textbooks and application papers. These comprehensive literature resources also addressed the computational models of the immune system and their applications till 1998. Various modeling techniques based on ordinary differential equations, delay differential equations, partial differential equations, agent-based models, stochastic differential equations as well as associated algorithm and simulation frameworks are being used to simulate IS [21].

This diversity of AIS knowledge has helped the researchers to apply AIS to solve several bench-mark problems of the field such as; computer security, numerical function optimization, combinatorial optimization, learning, bioinformatics, image processing, robotics, adaptive control system, data mining, and anomaly or error diagnosis [22]. As a more advance innovation, the applications of AIS in controlling a robotic arm is evident from studies, remote-sensing classification of satellite images, compensating exposure in images with back-lighting, webmining applications and application in industrial manufacturing process [23-26].

A review of literature has concluded the application of AIS in following three major categories as shown below. However, the newly identified and explored areas may fall beyond these categories Figure 3 [11,23,27-29].

\section{AIS Application in Robotics}

AIS can provide a baseline for robots to learn new skills, adapt to new environment throughout its lifetime. With the progress in mechatronics, MEMS (Micro-Electro Mechanical Systems) and nanotechnology the sizes and cost of electronic components (e.g. sensors, actuators and electronic boards) are decreasing, thus robots built from such components if produced at larger size will be very cheap. It would be possible to install/deploy a large number of such small robots and should rightly be called swarm of robots to achieve the desired task. AIS will play its role by bringing intelligence into this swarm of robots to achieve/accomplish the desired task. This idea has been specifically taken from Immune cells of adaptive or innate immunity. In this regard, the behaviour of neutrophils is particularly the interest of author with its potential application in Swarm Robotics.

Neutrophils are the most abundant white blood cells as constitute about $40-70 \%$ of the white blood cells in the blood stream [30]. As a first defense line, they respond early to threats against the hosts by detecting changes in the vascular endothelium induced by tissue damage and/or infection [31]. The behaviour of Neutrophils along with other immune cells is quite complex and through the microvascular system initially get access to the source of antigen. This causes changes in the vascular endothelium, giving impetus to neutrophils to exit the microvasculature and to move through the tissue by sensing molecules, or chemokines, produced by damaged or infected tissue $[31,32]$.

Thus, at the initial phase of inflammation caused by bacterial infection, environmental exposure or by some cancers, the first defensive response is received from the neutrophils which migrate towards the site of inflammation. Neutrophils first migrate through the blood vessels and then through the interstitial tissue, following chemical signals (Chemokines produced by damaged or infected tissues) such as Interleukin-8 (IL-8), C5a, fMLP and Leukotriene B4 in a process called "Chemotaxis" as indicated in Figure 4 [31-33].

This process, termed chemotaxis, plays a significant role in

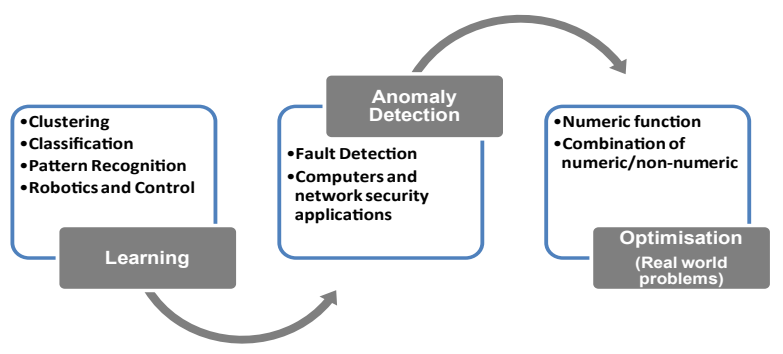

Figure 3: Categorical Areas for AIS Applications. 


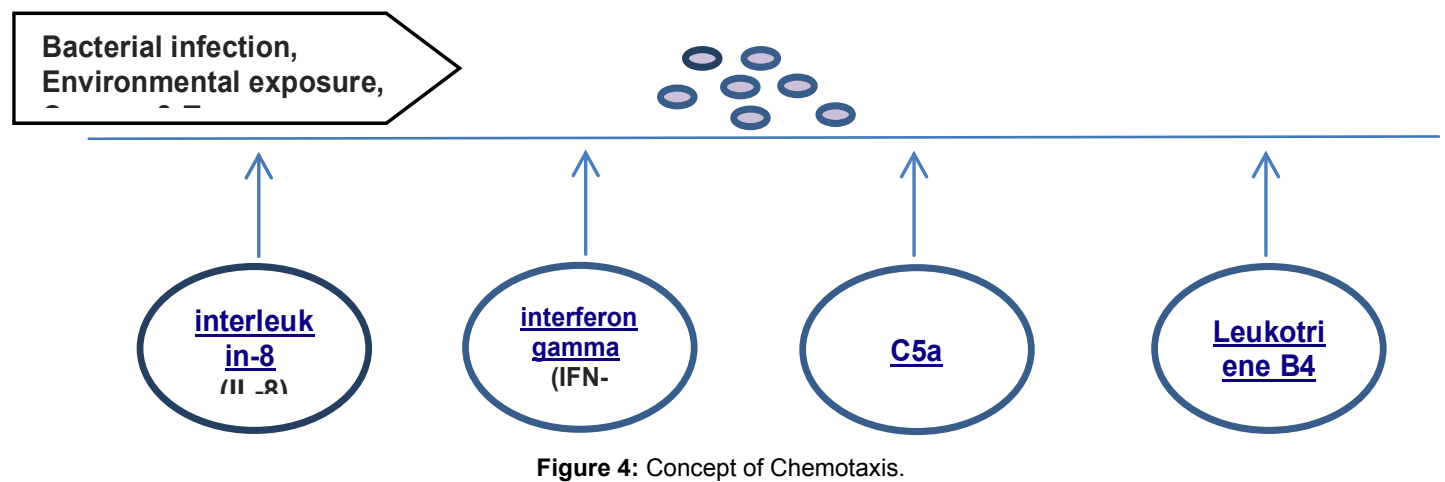

Figure 4: Concept of Chemotaxis.

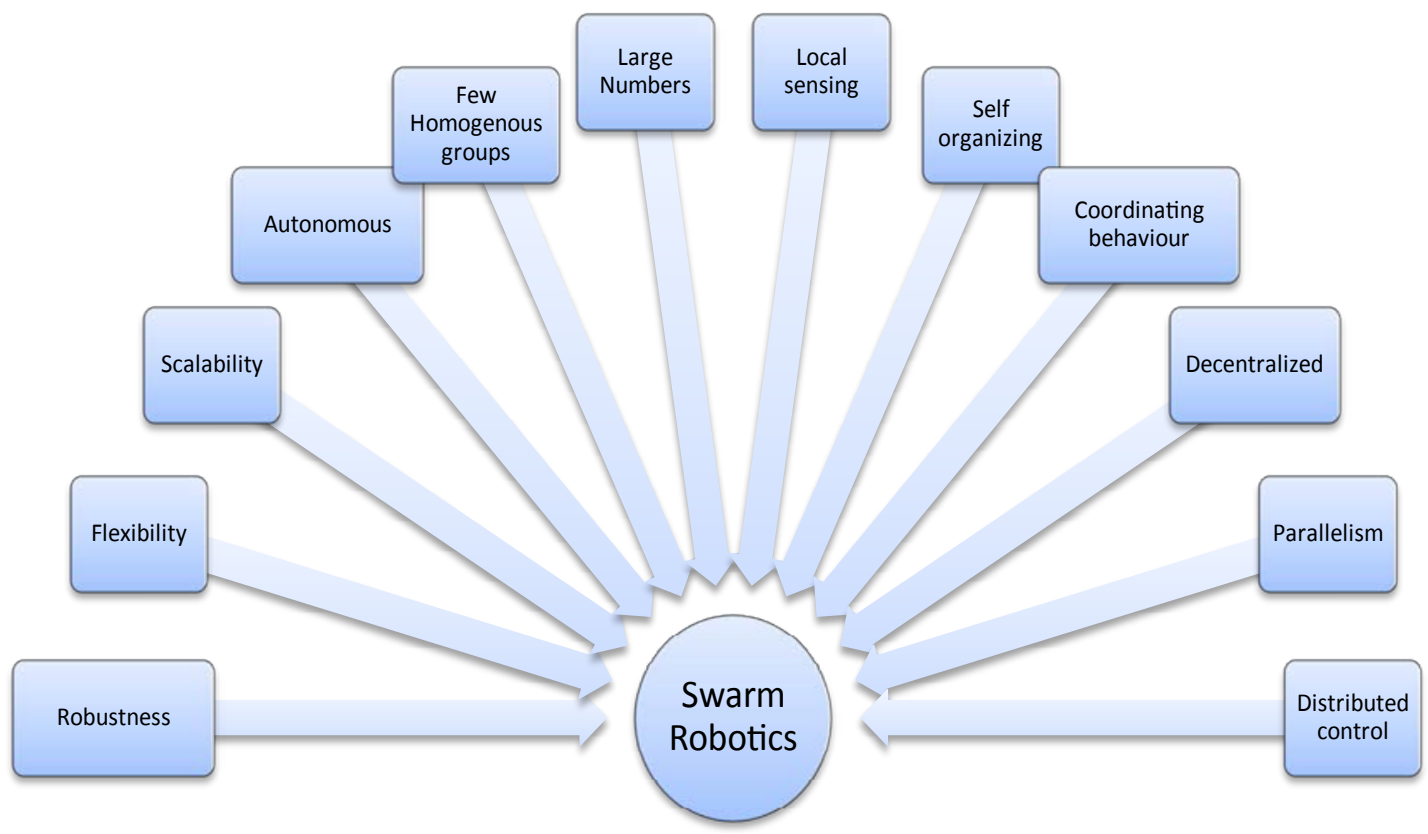

Figure 5: Characteristics of Swarm Robotics.

immune cell motion computation models [34]. These fundamental behaviours of neutrophils are mathematically well explained through the convection-diffusion and reaction-diffusion equations $[35,36]$.

The immune system swarms like neutrophil swarms initially develop from the primary arrest of a small count of neutrophils followed minutes later by massive numbers. This directed migration or dynamic swarming is probably caused by intercellular communication via signaling molecules. These signaling molecules are produced by, and are attracted to, neutrophils. Streaming is another dynamic behavior of neutrophils. It is assumed that neutrophils generate signals to induce swarming and once a swarm reaches a certain size, a large enough signaling center exists to overcome the competing signals of nearby smaller swarms [37].

\section{AIS Applications in Swarm Robotics}

Sahin (2005) has defined swarm robotics as "swarm robotics is the study of how large number of relatively simple physically embodied agents can be designed such that a desired collective behaviour emerges from the local interactions among agents and between the agents and the environment" [38]. Millonas has also proposed that a swarm system must encapsulate the principles of proximity, quality, diverse response, stability and the adaptability [39].

Swarm robotics is an innovative approach to the coordination of large numbers of robots. Basic motivation comes from the observation of birds/insects as to how these individual entities in itself can cooperate together to carry out complex tasks/goals that cannot be accomplished individually. This sort of coordination capabilities are still beyond the reach of current multi-agent robotic systems. The main characteristics that a swarm robotic system must possess are shown below as Figure 5.

The main feature of swarm behaviour is that each individual (robot) follows simple rules and there is no centralised control dictating their behaviour. Each robot is capable of observing and responding to its environment and directing its activity towards achieving common goal. Robots will collaborate through interaction among themselves exhibiting simple behaviours like self-assembly, self-repair, cooperation, monitoring and responding. 


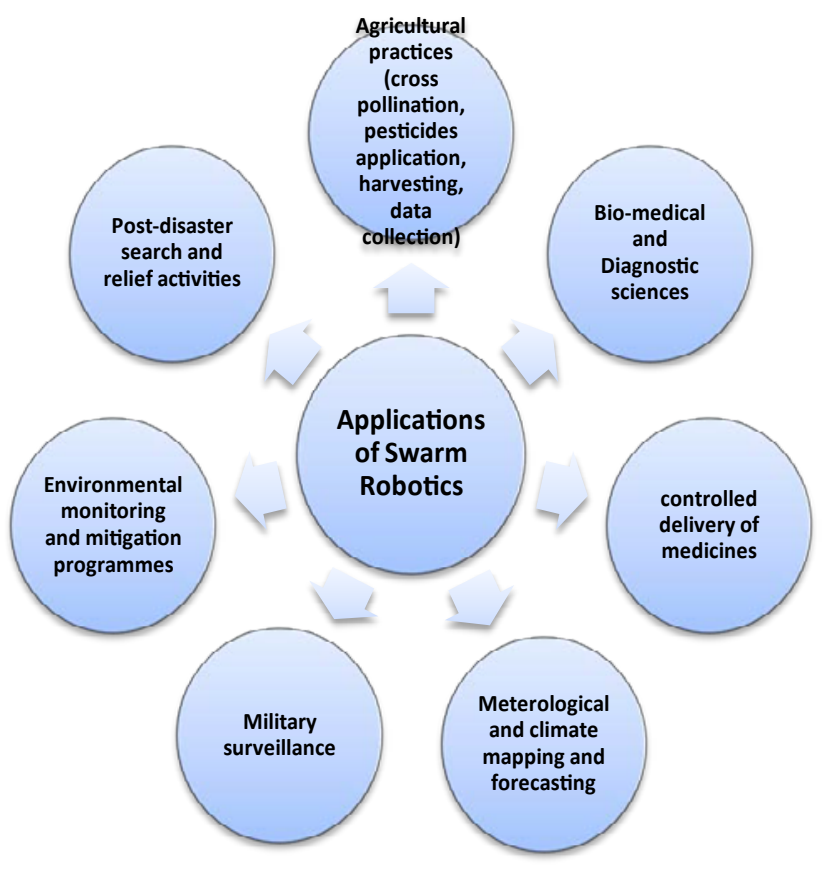

Figure 6: Application of Swarm Robotics in real world.

Following this, it is assumed that mathematical modelling, design and methodology of emergence as well as testing of swarm systems are key tasks to apply neutrophils behaviour in swarm robotics.

\section{Research Needs in AIS and Swarm Robotics}

Based on above facts, it is concluded that immune system is a very complex system and still there is a lack of reliable data about many of its constituent cells and molecules, and thus any simulation that intends to model the immune system at full scale will require huge computational resources. Thus, it allows the interconnection of predictive models, defined at multiple scales (molecules, cells, tissues and organs, bodywide systems, the whole organism, and collection of organisms) for conversion into in systemic networks. These networks can address and validate the systemic hypotheses by combining clinical observations, experimentation and predictive modelling. AIS based on neutrophils behavior, will play potential role by bringing intelligence into swarm of robots. An extensive literature review has concluded that Swarm Robotics has high prospects and utilization for a variety of challenging applications as shown in the Figure 6 below.

Application of swarm robotics in wide range of real life fields cannot be ignored for serious consideration for further developments. These applications include biomedical applications for developing micronano systems by applying high-resolution monitoring, fast prototyping of micro-environments such as by application of micro-nano robots may be for on-site drug delivery, development of unconventional robots (for DNA devices, nano-particles, synthetic bacteria, bio-bots for advance treatments), synthetic biology applications based on fabrication and manipulation at the micro-nano scale, development of energy-based robot control (magnetic, light-based self-organizing biological and robotic systems) and finally the control of swarm robotic systems with large-scale simulations will be applicable to all such applications. This can be further explored for the development of tools to explore swarm behaviours (bio-inspiration, crowdsourcing, machine learning).

Swarm robots can disperse and perform multiple tasks at difficult and inaccessible sites such as in forests, lakes, hilly areas etc. Swarms of robots because of the robustness of the swarm, can prove highly useful for dangerous tasks including monitoring and mitigating the environmental hazards, like a leakage of a chemical substance, clearing off environment from hazardous wastes.

Environmental pollution is one of the biggest concerns of planet earth in context to survival of all living organisms and thus application of AIS and SR in environmental monitoring and mitigation may prove most beneficial, if applied precisely.

Dramatic incidences of aquatics pollution due to oil spills, organic pollutants and suspended matters in surface water bodies such as oceans, rivers, lakes etc. have highlighted the potential to address the man-made environmental damage. Mitigation of this unwanted situation requires resources in terms of man power, machinery, labour cost and time. Hence, to provide alternative to all such requirements and constraints, research based on application of advance computational approaches is required to address this real world problem of environmental pollution.

Another environmental application may be based on developing in-pipe inspection swarm robots capable to overcome the issues of human factor in labour and time intensive monitoring and also to act in inaccessible environment inside the water or gas pipelines of an area within shortest possible time for long-distance inspection.

Future research is required to investigate deployment possibility of swarm of robots to move on the water's surface autonomously and gather organic pollutants and suspended matters to preserve water resources from quality deterioration. The robots may work together to cover a large area of the water and communicate with one another and with land-bound researchers. This will also help in developing an insight so as to how the problems such as large scale simulation, control of mirco/nano swarm robots can be dealt with by using AIS.

\section{References}

1. Castro LNde, Zuben FJV (1999) Artificial Immune Systems: Part I -Basic Theory and Applications. School of Computing and Electrical Engineering, State University of Campinas, Brazil.

2. Grasso P, Gangolli S, Gaunt I (2002) Essentials of Pathology for Toxicologists $\left(1^{\mathrm{ST}} \mathrm{Ed}\right) \mathrm{CRC}$ Press.

3. Alberts B, Alexander J, Julia L, Martin R, Keith R, et al. (2002) Molecular Biology of the Cell. (4th Ed). New York and London, Garland Science.

4. Kephart JO (1994) A biologically inspired immune system for computers artificial life IV. In: Brooks RA, Maes P (Eds), Proceedings of 4th International Workshop on the Synthesis and Simulation of Living Systems, MIT Press.

5. Dasgupta D (1996) Using immunological principles in anomaly detection. In: Proceedings of the Artificial Neural Networks in Engineering (ANNIE96), St Louis, USA.

6. Forrest S, Perelson AS, Allen L, Cherukuri R (1994) Self-nonself discrimination in a computer In: SP 1994: Proceedings of the 1994 IEEE Symposium on Security and Privacy, IEEE Computer Society, Washington, DC.

7. Dasguptaa D, Yua S, Nino F (2011) Recent Advances in Artificial Immune Systems: Models and Applications. Applied Soft Computing 11: 1574-1587.

8. Dasgupta D (2006) Advances in artificial immune systems. Computational Intelligence Magazine, IEEE.

9. Somayaji A, Hofmeyr S, Forrest S (1997) Principles of a Computer Immune System. In: Proceedings of the Second New Security Paradigms Workshop 75-82 
Citation: Daudi J (2015) An Overview of Application of Artificial Immune System in Swarm Robotic Systems. Adv Robot Autom 4: 127. doi: 10.4172/21689695.1000127

10. Burnet FM (1959) The Clonal Selection Theory of Acquired Immunity. Cambridge University Press.

11. Castro LND, Zuben FJV (2000) The clonal selection algorithm with engineering applications, Genetic and Evolutionary Computation Conference (GECCO'00) - Workshop Proceedings, Las Vegas, Nevada, USA.

12. Ishida $Y$ (1990) Fully distributed diagnosis by PDP learning algorithm: towards immune network PDP model, IEEE International Joint Conference on Neural Networks, San Diego, USA.

13. Knight T, Timmis J (2001) AINE: an immunological approach to data mining, In: IEEE International Conference on Data Mining, San Jose, CA, USA.

14. Matzinger $P$ (2002) The danger model: are Renewed sense of self. Science 296: 301-305.

15. Matzinger $P$ (1994) Tolerance,danger, and the extended family. Annu.Rev. Immunol 12: 991-1045.

16. Greensmith J, Aickelin J, Cayzer S (2008) Detecting Danger: The Dendritic Cell Algorithm.Copyright Robust Intelligent systems, IGI Publishing.

17. Dasgupta D, Yu S, Majumdar NS (2003) MILA - multilevel immune learning algorithm, in: Genetic and Evolutionary Computation Conference (GECCO 2003), Chicago, IL, USA.

18. Aickelin U, Cayzer S (2002) The danger theory and its application to artificia immune systems. The 1st International Conference on Artificial Immune Systems (ICARIS 2002), Canterbury, England.

19. Greensmith J, Aickelin U, Cayzer S (2005) Introducing dendritic cells as a novel immune-inspired algorithm for anomaly detection. Artificial Immune Systems Lecture Notes in Computer Science 3627: 153-167.

20. Yus S, Dasgupta D (2008) Conserved Self Pattern Recognition Algorithm Artificial Immune Systems Lecture Notes in Computer Science Volume 5132 279-290.

21. Kim H (2009) Asymptotic problems for stochastic processes and related differential equations. PhD Dissertation. Department of Mathematics. University of Maryland, USA.

22. Hart E, Timmis J (2008) Application areas of AIS: the past, present and future. Journal of Applied Soft Computing, 8: 191-201.

23. Lee Z J, Lee CY, Su SF (2002) An immunity based ant colony optimization algorithm for solving weapon-target assignment problem. Appl. Soft Comput 2: 39-47.

24. Zhong Y, Zhang L, Huang B, Li P (2006) An unsupervised artificial immune classifier for multi/hyperspectral remote sensing imagery. IEEE Trans. Geosci. Remote Sens 44: 420-431.
25. Nasraoui O, Rojas C, Cardona C (2006) A framework for mining evolving trends in web data streams using dynamic learning and retrospective validation. Comput. Networks 50: 1488-1512.

26. Bailey S (1984) From desktop to plant floor, a CRT is the control operators window on the process. Control Engineering 31: 86-90.

27. Campelo F, Guimarães FG, Igarashi H, Ramírez J, Noguchi S (2006) A modified immune network algorithm for multimodal electromagnetic problems. IEEE Transactions on Magnetics 42.

28. Kalini A, Karaboga N (2005) Artificial immune algorithm for iir filter design. Eng. Appl. Artif.Intell 18: 919-929.

29. Castro LND, Zuben FJV (2002) Learning and optimization using the clonal selection principle. IEEE Transactions on Evolutionary Computation 6: 239251.

30. Beers MH, Porter RS, Jones TV (2006) The Merck Manual. (18 ${ }^{\text {th }}$ Ed), Merck and Co., Inc.

31. Janeway CA, Travers P (1997) Immunobiology: The Immune System in Health and Disease. Churchill Livingstone, New York.

32. Ariel A, Serhan NC (2007) Resolvins and protectins in the termination program of acute inflammation, Trends Biochem. Sci. 28: 176-183.

33. Kadirkamanathan V, Anderson SR, Billings SA, Zhang X, Holmes, et al. (2012) The Neutrophil's Eye-View: Inference and Visualisation of the Chemoattractant Field Driving Cell Chemotaxis In Vivo. PLoS One 7: e35182.

34. Nathan C (2006) Neutrophils and immunity: Challenges and opportunities. Nat Rev. Immunol. 6:173-182.

35. Holmes GR, Dixon G, Anderson SR (2012) Drift-Diffusion Analysis of Neutrophil Migration during Inflammation Resolution in a Zebrafish Model. Advances in Hematology 8

36. Sua B, Zhoua W, Dormanb KS, Jones DE (2009) Mathematical modelling of immune response in tissues. Computational and Mathematical Methods in Medicine 10: 9-38.

37. Chtanova T, Schaeffer M, Han S, van Dooren G, Nollmann M (2008) Dynamics of Neutrophil Migration in Lymph Nodes during Infection. Immunity 29: 487-496.

38. Sahin E (2005) Swarm robotics: from sources of inspiration to domains of application. Swarm Robotics 3342: 10-20.

39. Millonas MM (1994) Swarms, phase transitions, and collective intelligence. In: Artificial life III. Addison-Wesley, Reading 417-445. 